\title{
Morfología Ósea de Sínfisis Mandibular. Estudio Piloto
}

\author{
Osseous Morphology in the Mandibular Symphysis. A Pilot Study
}

Humberto Velásquez ${ }^{*, * *}$; Sergio Olate ${ }^{*, * * *}$; Mariano del Sol ${ }^{* * * * *}$; Mario Cantín ${ }^{*, * * * *, * * * * * *}$ \& Márcio de Moraes ${ }^{* * * * * * *}$

VELÁSQUEZ, H.; OLATE, S.; DEL SOL, M.; CANTIN, M.; DE MORAES, M. Morfología ósea de sínfisis mandibular. Estudio piloto. Int. J. Morphol., 33(1):355-360, 2015.

RESUMEN: El objetivo de este estudio fue evaluar la relación de tejido óseo cortical y esponjoso en el sector anterior de la mandíbula. Se seleccionaron 14 sujetos de ambos sexos para realizar el estudio piloto de este protocolo; en todos los sujetos se realizó un estudio con tomografía computarizada de haz cónico para realizar análisis morfométrico en base a la posición de los dientes canino, incisivo lateral e incisivo central de ambos lados; se analizó la distancia vertical desde el ápice dentario hasta el punto más inferior del margen mandibular y se analizó la distancia anteroposterior en dos niveles inferiores del ápice (5 mm y $10 \mathrm{~mm}$ ); en estos niveles se identificó el tamaño de hueso cortical y el tamaño de hueso esponjoso. Se observó que el hueso cortical es de mayor tamaño en la cortical lingual que la cortical bucal, con casi $1 \mathrm{~mm}$ de diferencia; el hueso esponjoso fue similar a la sumatoria de ambos huesos corticales (5 mm aproximadamente) en las áreas evaluadas. Se concluye que el hueso cortical es menor en el sector bucal al compararse con el lingual; la distancia vertical desde el ápice dentario hasta el margen mandibular fue de 16,5 y 21,05 mm.

PALABRAS CLAVE: Sínfisis mandibular; Morfología mandibular; Osteotomía mandibular.

\section{INTRODUCCIÓN}

La región anterior de mandíbula ha tomado interés en los últimos años, debido a la variedad de procedimientos quirúrgicos que son desarrollados en ella. La cirugía estética facial mediante osteotomías de reposición tridimensional del mentón (Olate \& De Moraes, 2014) y osteotomías para mejorar las condiciones respiratorias en la fase II del protocolo de Stanford (Powell \& Riley, 1995), son ejemplos claros de la necesidad de reconocer las condiciones anatómicas del sector para realizar procedimientos de baja morbilidad.

Existen limitados estudios que definan las condiciones de hueso cortical y esponjoso en la región anterior de mandíbula. Buena parte de las investigaciones se orientan a reconocer las condiciones de inervación, posición del foramen mental y las relaciones dentarias asociadas ( $\mathrm{Vu}$ et al., 2015). El reconocer las características óseas en sujetos dentados es prioritario para el estudio de diferentes opciones quirúrgicas utilizadas en esta área.
El estudio de imágenes tridimensionales es constantemente utilizado en el diagnóstico y planificación quirúrgica, transformándolas en elementos básicos para el estudio morfológico (Olate et al., 2012, 2014a). Ventajas importantes de este sistema para el análisis en morfología facial se asocian a la ausencia de modificaciones de la muestra (no existe necesidad de interrupción en muestras anatómicas) y al hecho de presentar una muestra anatómica integral donde se pueden asociar otras variables anatómicas del sector estudiado (Li et al., 2006). Además, es viable de identificar otras condicionantes como las deformidades faciales o la ausencia parcial dentaria en el análisis sectorial de la muestra.

El objetivo del presente estudio es presentar los datos preliminares del análisis morfológico de la estructura cortical y esponjosa en región anterior de la mandíbula.

\footnotetext{
* Programa de Magíster en Odontología, Facultad de Odontología, Universidad de La Frontera, Temuco, Chile.

** Facultad de Odontología, Universidad San Sebastián, Puerto Montt, Chile.

*** División de Cirugía Oral y Maxilofacial \& CIMA, Facultad de Odontología, Universidad de La Frontera, Temuco, Chile.

***** Centro de Investigación en Ciencias Biomédicas, Universidad Autónoma de Chile, Temuco, Chile.

****** Programa de Doctorado en Ciencias Morfológicas, Universidad de La Frontera, Temuco, Chile.

****** División de Cirugía Oral y Maxilofacial, Universidad Estadual de Campinas, Piracicaba, Brasil.
} 


\section{MATERIAL Y MÉTODO}

Se desarrolló un estudio descriptivo para analizar la estructura ósea anterior de la mandíbula. Se ingresaron sujetos que cumplieran requisitos de inclusión como no presentar: ausencias dentarias (a excepción del tercer molar), cirugías faciales, trauma facial previo, patología quística o tumoral en el sector anterior de la mandíbula, siendo todos ellos pacientes que accedieran voluntariamente al estudio. Se excluyeron sujetos que presentaban alteraciones de la morfología radicular en dientes del sector anteroinferior, sujetos con tratamiento endodóntico en el sector anteroinferior y sujetos con enfermedad periodontal severa con reabsorción ósea interproximal debido a que todas estas condicionantes podrían influenciar en la pérdida ósea en el sector anterior de la mandíbula.

El estudio se realizó analizando imágenes planas obtenidas de tomografía computarizada de haz cónico (TCCB) realizadas en posición de máxima intercuspidación utilizando el equipo PlanMeca® (Korea); las imágenes fueron analizadas en el programa computacional EZ3D plus (2009) por un observador único siguiendo protocolos de repetición de mediciones y análisis intra clase de rutina según el protocolo desarrollado.

Los puntos de medición se localizaron en relación a los dientes canino, incisivo lateral e incisivo central, del lado derecho e izquierdo en cada mandíbula; en todos estos dientes se plantearon 8 unidades de medición (expresadas en milímetros) definidas de la siguiente manera:

A - Distancia vertical: línea vertical que une el punto más apical del diente seleccionado hasta el punto más inferior de la cortical ósea inferior (Fig. 1).

B - Distancia Horizontal 1: línea horizontal que une el punto más anterior de la cortical ósea anterior y el punto más posterior de la cortical ósea posterior. Esta línea se desarro1la $5 \mathrm{~mm}$ bajo el ápice del diente seleccionado y forma un ángulo recto al trazarse sobre la línea A (Fig. 1).

C - Distancia Horizontal 2: línea horizontal que une el punto más anterior de la cortical ósea anterior y el punto más posterior de la cortical ósea posterior. Esta línea se desarrolla $10 \mathrm{~mm}$ bajo el ápice del diente seleccionado y forma un ángulo recto al trazarse sobre la línea A (Fig. 1).

D - Distancia Hueso Esponjoso 1: línea B considerando solamente el hueso esponjoso (Fig. 2).

E - Distancia Hueso Esponjoso 2: línea C considerando solamente el hueso esponjoso (Fig. 2).

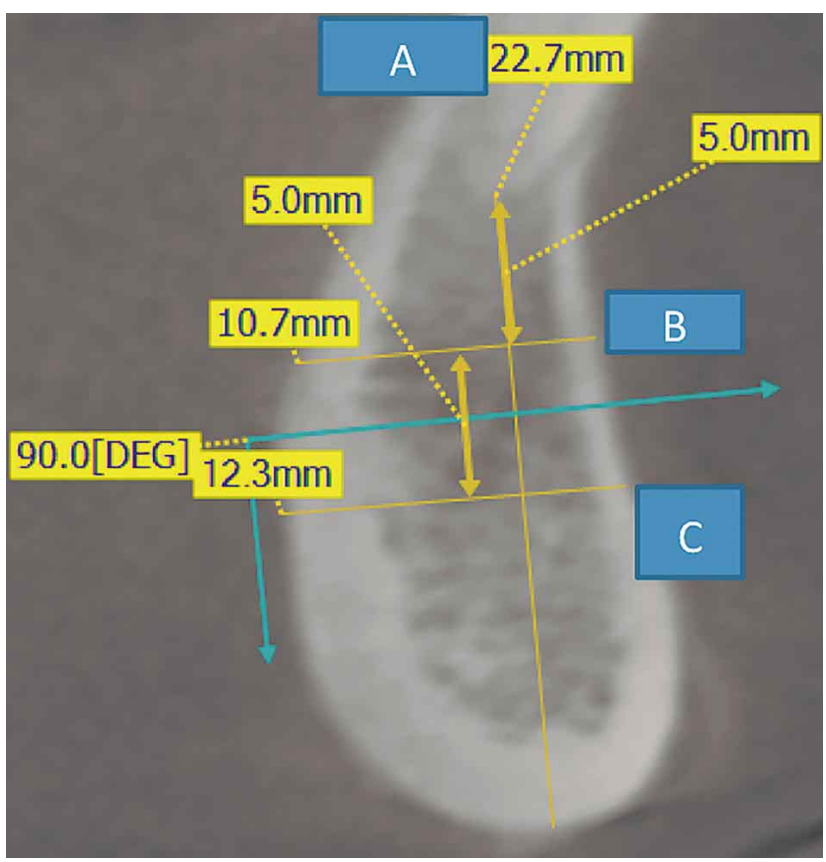

Fig. 1. Mediciones realizadas en la imagen sagital de la TCCB. En A se observa la medición vertical; en B y C se observan las mediciones horizontales, paralelas entre sí.

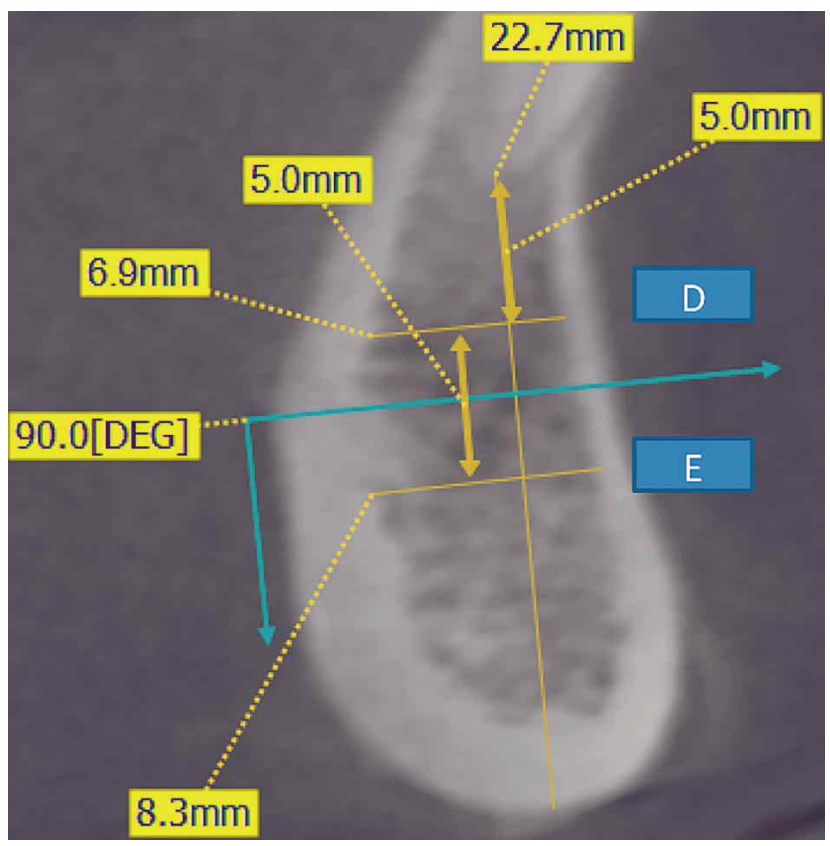

Fig. 2. Mediciones realizadas en la TCCB de los 14 pacientes analizados donde $\mathrm{D}$ y E corresponden a la medición del hueso esponjoso de las líneas previas B y C.

F - Distancia Hueso Cortical A1: línea B considerando solamente el hueso cortical de la cortical anterior (Fig. 3).

G - Distancia Hueso Cortical A2: línea C considerando solamente el hueso cortical de la cortical anterior (Fig. 3). 
H - Distancia Hueso Cortical Inferior: línea A considerando solamente el hueso cortical de la cortical inferior (Fig. 3). I - Distancia Hueso Cortical P2: línea C considerando solamente el hueso cortical de la cortical posterior (Fig. 3).

J - Distancia Hueso Cortical P1: línea B considerando solamente el hueso cortical de la cortical posterior (Fig. 3).

Los resultados se analizaron de forma descriptiva, realizando un seguimiento comparativo entre los dientes analizados para relacionarlos con la literatura existente.

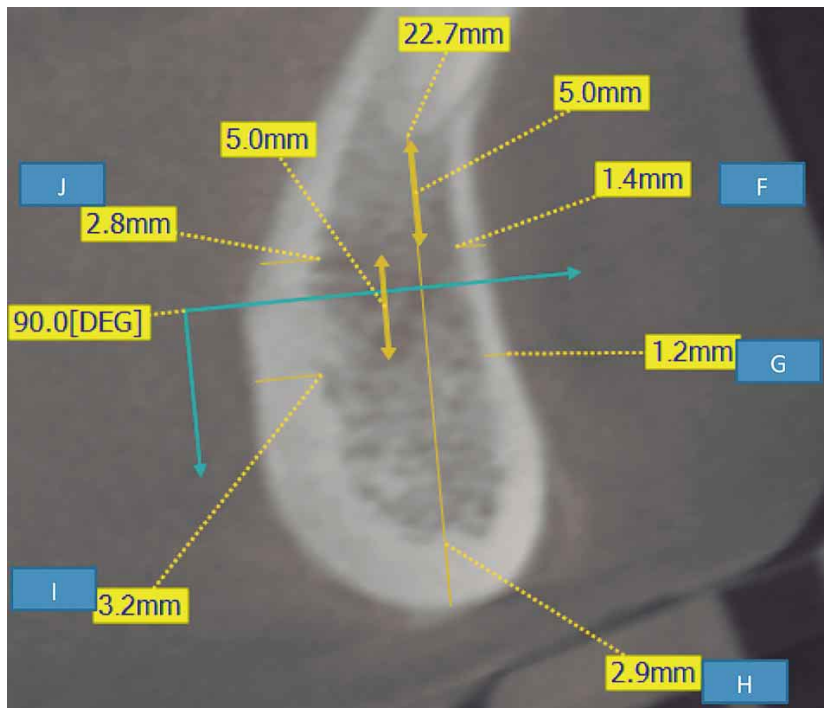

Fig. 3. Mediciones realizadas en la TCCB identificando las valoraciones de tejido cortical, los cuales son obtenidos desde las líneas horizontal y vertical inicial.

\section{RESULTADOS}

Los resultados se presentan en las Tablas I, II y III. Fue observado que en las mediciones de mayor extensión, la vertical (A) y las dos horizontales (B y C) existían valores promedios cercanos, sin diferencias mayores a $1 \mathrm{~mm}$ entre ambas mediciones. La misma situación se observó en las mediciones de tejido esponjoso.

En la medición vertical se estableció que la distancia vertical del canino (línea A) esta entre los promedios de 17,02 y $16,5 \mathrm{~mm}$; a nivel de incisivo lateral, estos registros fueron de 18,8 y $20,01 \mathrm{~mm}$ y a nivel del incisivo central fueron de 21,05 y $20,97 \mathrm{~mm}$, lo cual muestra diferencias de $4 \mathrm{~mm}$ de entre las mediciones verticales de caninos e incisivos.

Las mediciones horizontales a nivel superior (línea B) se observaron dentro de rangos de 8,41 y $9,12 \mathrm{~mm}$, indicando diferencias menores de $0,8 \mathrm{~mm}$ entre los valores obtenidos tanto en caninos como incisivos. El sector de la horizontal inferior (línea C) mostró mayor variedad estableciendo rangos de entre 9,38 y $11,91 \mathrm{~mm}$, siendo en el sector de canino el que presentaba los registros de menor tamaño.

Con respecto al tejido esponjoso, siempre existió un mayor tamaño de tejido esponjoso en el sector inferior (línea E) al compararlo con el sector superior; los menores valores de tejido esponjoso se observaron en el sector de caninos. En el análisis del hueso cortical, se observó que la cortical inferior (área del margen mandibular) fue mayor a las otras áreas de hueso cortical medidas; en términos gene-

Tabla I. Mediciones horizontales y vertical registradas para cada diente analizado (unidad de medición en mm).

\begin{tabular}{lcccc}
\hline \multirow{2}{*}{ Diente } & \multicolumn{3}{c}{ Medición (Promedio \pm Desviación Estándar, mm) } \\
\cline { 3 - 5 } & & A & B & C \\
\hline \multirow{3}{*}{ Derecho } & Canino & $17,02( \pm 2,16)$ & $8,55( \pm 2,19)$ & $9,97( \pm 1,98)$ \\
& I. Lateral & $18,8( \pm 5,57)$ & $8,82( \pm 1,91)$ & $10,56( \pm 1,52)$ \\
& I. Central & $21,05( \pm 3,25)$ & $8,94( \pm 2,43)$ & $11,66( \pm 1,55)$ \\
\multirow{3}{*}{ Izqueirdo } & I. Central & $20,97( \pm 2,87)$ & $8,84( \pm 2,48)$ & $11,91( \pm 1,76)$ \\
& I. Lateral & $20,01( \pm 2,95)$ & $9,12( \pm 2,81)$ & $10,42( \pm 1,96)$ \\
& Canino & $16,5( \pm 3,01)$ & $8,41( \pm 2,29)$ & $9,38( \pm 2,21)$ \\
\hline
\end{tabular}

Tabla II. Mediciones horizontales de tejido esponjoso registradas para cada diente analizado (unidad de medición en mm).

\begin{tabular}{lccc}
\hline \multirow{2}{*}{ Diente } & \multicolumn{2}{c}{ Medición (Promedio \pm Desviación Estándar, mm) } \\
\cline { 3 - 4 } \multirow{2}{*}{ Derecho } & D & E \\
\cline { 3 - 4 } \multirow{4}{*}{ Izqueirdo } & Canino & $5,03( \pm 1,65)$ & $5,01( \pm 2,17)$ \\
& I. Lateral & $4,89( \pm 1,84$ & $6,07( \pm 1,89)$ \\
& I. Central & $5,17( \pm 1,77)$ & $6,61( \pm 1,45)$ \\
& I. Central & $5,47( \pm 2,09)$ & $6,9( \pm 1,72)$ \\
& I. Lateral & $5,06( \pm 1,95)$ & $5,88( \pm 1,52)$ \\
& Canino & $4,76( \pm 1,7)$ & $3,97( \pm 2,11)$ \\
\hline
\end{tabular}


Tabla III. Mediciones del hueso cortical correspondientes a las mediciones horizontal y vertical registradas para cada diente analizad (unidad de medición en mm).

\begin{tabular}{lcccccc}
\hline \multirow{2}{*}{ Diente } & \multicolumn{4}{c}{ Medición (Promedio \pm Desviación Estándar, mm) } \\
\cline { 3 - 6 } & & F & G & H & I & J \\
\hline \multirow{3}{*}{ Derecho } & Canino & $1,52( \pm 0,35)$ & $1,87( \pm 0,34)$ & $3,62( \pm 1,08)$ & $3,16( \pm 0,57)$ & $2,12( \pm 0,51)$ \\
& I. Lateral & $1,46( \pm 0,26)$ & $1,66( \pm 0,36)$ & $2,84( \pm 0,54)$ & $3,12( \pm 0,81)$ & $2,63( \pm 0,62)$ \\
& I. Central & $1,52( \pm 0,36)$ & $1,98( \pm 0,65)$ & $2,54( \pm 0,73)$ & $3,1( \pm 0,64)$ & $2,47( \pm 0,60)$ \\
\multirow{3}{*}{ Izqueirdo } & I. Central & $1,45( \pm 0,24)$ & $1,87( \pm 0,40)$ & $2,83( \pm 1,52)$ & $3,12( \pm 0,65)$ & $2,68( \pm 0,64)$ \\
& I. Lateral & $1,44( \pm 0,33)$ & $1,76( \pm 0,45)$ & $3,08( \pm 0,97)$ & $2,9( \pm 0,71)$ & $2,76( \pm 1,1)$ \\
& Canino & $1,67( \pm 0,34)$ & $2,03( \pm 0,32)$ & $3,9( \pm 1,49)$ & $3,08( \pm 0,88)$ & $2,21( \pm 0,73)$ \\
\hline
\end{tabular}

rales, la cortical anterior en todos los dientes evaluados se observó entre 1,44 y 1,67 mm, mientras que la cortical posterior presentó valores de entre 2,12 y 3,16 mm, siendo menor los valores obtenidos en las mediciones de caninos.

\section{DISCUSIÓN}

Esta investigación tuvo como objetivo reconocer las características óseas de la región anterior de mandíbula en su fase preliminar y orientar nuevas investigaciones en morfología de mandíbula debido a que procedimientos quirúrgicos que afectan esta área son cada vez más frecuentes y requieren de mayor atención toda vez que la morbilidad asociada puede aumentar si no son observados los criterios anatómicos aquí descritos. En térmicos generales, se observó valores adecuados en las relaciones de tejido cortical y tejido esponjoso. En el rango de las mediciones de la línea $\mathrm{B}$ y C se observó que la cortical lingual es casi $1 \mathrm{~mm}$ de mayor tamaño al compararla a la cortical anterior; al sumar ambas estructuras corticales, anterior y posterior de cada línea (B ó C) se identificó que el hueso esponjoso es casi equivalente al tamaño de ambas corticales.

Flanagan (2008) reportó que en sujetos edéntulos, el hueso cortical lingual fue de $2,33 \mathrm{~mm}$ mientras que el bucal fue de $1,79 \mathrm{~mm}$, lo cual se asemeja a nuestros resultados toda vez que mantiene la tendencia de que el sector lingual es aproximadamente $1 \mathrm{~mm}$ mayor que el sector bucal. Semejantes resultados fueron observados por Kim et al. (2012) quienes estudiaron cadáveres dentados concluyendo el mayor tamaño del hueso cortical lingual. En este mismo sentido Genisa et al. (2014) indicaron que en 10 sujetos evaluados con tomografías de haz cónico se observó que el hueso cortical lingual fue significativamente mayor al hueso cortical bucal, presentando diferencias de 1, $34 \mathrm{~mm}$ en el sector anterior y 2,92 en el sector posterior (1,58 $\mathrm{mm}$ de diferencia).

Las consideraciones clínicas de esta área se han centrado en algunos procedimientos quirúrgicos (Cáceres et al., 2014), como la toma de injertos óseos donde tradicional- mente se indicó que $5 \mathrm{~mm}$ desde cualquier otra estructura anatómica seria suficiente para disminuir la morbilidad en la captura del injerto (Hunt \& \& Jovanovic, 1999). Sin embargo, Pommer et al. (2008) evaluaron 50 tomografías computadorizadas de mandíbulas dentadas concluyendo que los márgenes de seguridad se realizarían cuando la osteotomía es anterior en $5 \mathrm{~mm}$ al foramen mental, $8 \mathrm{~mm}$ bajo el ápice dentario y cuando la profundidad de la osteotomía no supera los $4 \mathrm{~mm}$; en base a este último dato y basado en nuestro reporte, la toma de injerto correspondería esencialmente a hueso cortical más $1 \mathrm{~mm}$ de hueso esponjoso, lo cual es clínicamente importante en la toma de decisiones para la obtención de injertos autógenos.

En este sentido, Brockhoff et al. (2014) estudiaron en cadáveres la condición anatómica en la toma de injertos óseos obtenidos de sínfisis mandibular y señalaron que el volumen óseo obtenido en esta área no presentaba diferencias entre hombres y mujeres o entre sujetos dentados o edéntulos; señalaron, además, que existían diferencias en el tamaño de hueso cortical vestibular en sujetos con o sin dientes, siendo mayor en los sujetos dentados. Dentro de las limitaciones observadas en la literatura disponibles que no se consideran las características mandibulares de sujetos con anomalías esqueletales (deformidad dentofacial) (Muñoz et al., 2014; Olate et al., 2014b) y no son integrados en los análisis desarrollados; de esta manera, indicar el volumen promedio de tejido óseo que puede ser retirado en un determinado sector anatómico es variable en base a la condición esqueletal del sujeto. Joshi et al. (2014) determinó diferencias morfológicas en situación espacial y morfología máxilo mandibular en sujetos con deformidades faciales, transformándolo en un elemento importante para definir condiciones de la morfología asociada. Baysal et al. (2013) indicaron diferencias significativas entre sujetos clase I y clase II dentaria al comparar el tamaño sagital de la cortical ósea en tomografía computarizada; ellos confirmaron que los sujetos clase II presentan menor tamaño óseo de la cortical anterior en áreas próximas al diente. También, Sharma et al. (2012), indicaron que el tamaño de la cortical ósea bucal seria asociada al modelo de crecimiento craneofacial, estableciendo relaciones entre el tipo de deformidad facial y las características del hueso cortical. 
El hueso cortical entrega estabilidad a las fijaciones realizadas, haciéndolo necesario en procedimientos que requieran implantes dentales y también en la reducción y osteosíntesis de fracturas, osteotomías programadas para reposición mandibular, distracción osteogénica mandibular o la instalación de implantes dentales (Miyamoto et al., 2005)
En base a este estudio piloto se puede concluir que el hueso cortical bucal presenta menor tamaño cuando se compara al hueso cortical bucal. El hueso esponjoso presenta un tamaño próximo al obtenido por la suma de ambos huesos corticales lo cual puede indicar una relación de estabilidad. Los nuevos protocolos de estudios morfológicos deben incluir análisis según al clase facial del sujeto analizado.

VELÁSQUEZ, H.; OLATE, S.; DEL SOL, M.; CANTIN, M.; DE MORAES, M. Osseous morphology in the mandibular symphysis. A pilot study. Int. J. Morphol., 33(1):355-360, 2015.

SUMMARY: The aim of this research was to evaluate the relation between the cortical and cancellous bone in the anterior area of the mandible. Were selected 14 subjects, male and female, to make the pilot study with this protocol; in all of them was realized a cone beam computed tomography to make a morphometric analysis in agreed with de canine, lateral incisor and central incisor of the both, right and left, side. Was analyzed the distance between the apex of the tooth to the inferior point of the cortical bone in the marginal area and in 5 and $10 \mathrm{~mm}$ lower of the dental apex. In these levels were identified the cortical bone size and cancellous bone size. Was observed that the cortical bone is thicker in the lingual side than the buccal side (1 mm diference approximately); the cancellous bone size is like the lingual and buccal cortical size (5 $\mathrm{mm}$ approximately) in the evaluated areas. Its concluded that the buccal cortical bone is lower than lingual cortical bone; the vertical distance between the apex and the basilar area is close to 16.5 and $21.05 \mathrm{~mm}$.

KEY WORDS: Mandible symphysis; Mandible morphology; Mandible osteotomy.

\section{REFERENCIAS BIBLIOGRÁFICAS}

Baysal, A.; Ucar, F. I.; Buyuk, S. K.; Ozer, T. \&Uysal, T. Alveolar bone thickness and lower incisor position in skeletal class I and class II malocclusions assessed with cone-beam computed tomography. Korean J. Orthod., 43(3):134-40, 2013.

Brockhoff, H. C. 2nd.; Yates, D. M.; Finn, R. \& Phillips, C. Comparison of intraoral harvest sites in the edentulous versus dentate specimen. Oral Surg. Oral Med. Oral Pathol. Oral Radiol., 117(5):575-80, 2014.

Cáceres, F.; Ramírez, V. \& Soto, R. Influence of remnant teeth in the presence and morphometry of foramina and canals in relation to mental spine. Int. J. Morphol., 32(1):106-11, 2014.

Flanagan, D. A Comparison of facial and lingual cortical thicknesses in edentulous maxillary and mandibular sites measured on computerized tomograms. J. Oral Implantol., 34(5):256-8, 2008 .

Genisa, M.; Rajion, Z.; Pohchi, A.; Yap, J. \& Rafiq, M. Preoperative bone assessment using cone beam computed tomography. Int . Med. J., 21(4):412-5, 2014.

Hunt, D. R. \& Jovanovic, S. A. Autogenous bone harvesting: A chin graft technique for particulate and monocortical bone blocks. Int. J. Periodontics Restorative Dent., 19(2):165-73, 1999.

Joshi, N.; Hamdan, A. M. \& Fakhouri, W. D. Skeletal malocclusion: a developmental disorder with a life-long morbidity. J. Clin. Med. Res., 6:399-408, 2014.
Kim, H. J.; Yu, S. K.; Lee, M. H.; Lee, H. J.; Kim, H. J. \& Chung, C. H. Cortical and cancellous bone thickness on the anterior region of alveolar bone in Korean: a study of dentate human cadavers. J. Adv. Prosthodont., 4(3):146-52, 2012.

Li, T.; Schreibmann, E.; Yang, Y. \& Xing, L. Motion correction for improved target localization with on board cone-beam computed tomography. Phys. Med. Biol., 51(2):253-67, 2006.

Miyamoto, I.; Tsuboi, Y.; Wada, E.; Suwa, H. \& Iizuka, T. Influence of cortical bone thickness and implant length on implant stability at the time of surgery--clinical, prospective, biomechanical, and imaging study. Bone, 37(6):776-80, 2005.

Muñoz, G.; Olate, S.; Cantín, M.; Vásquez, B. \& Del Sol, M. TMJ in facial class III deformity. Condylar morphology relations. Int. J. Clin. Exp. Med., 7(9):3113-7, 2014.

Olate, S.; de Moraes, P. H.; Caria, P. H. F.; Netto, H. D. M. C. \& Barbosa, A. R. A. Craniofacial morphometry for craniofacial implant. Int. J. Morphol., 30(3):1166-72, 2012.

Olate, S.; Cantín, M.; Vásquez, B.; del Sol, M. \& de Moraes, M. Facial Soft Tissue in Subjects with Class II and Class III Facial Deformities: Preliminary Results. Int. J. Morphol., 32(3):1022-5, 2014a.

Olate, S.; Cantín, M.; Vásquez, B.; del Sol, M.; Henriquez-Alarcón, M. \& de Moraes, M. Pharyngeal Airway Space in Subjects with Class II and Class III Facial Deformities. Int. J. Morphol., 32(4):1271-6, 2014b. 
VELÁSQUEZ, H.; OLATE, S.; DEL SOL, M.; CANTIN, M.; DE MORAES, M. Morfología ósea de sínfisis mandibular. Estudio piloto. Int. J. Morphol., 33(1):355-360, 2015.

Olate, S. \& de Moraes, M. Cirugía facial estética: contribución de la osteotomía anterior de mandibular. Int. J. Med. Surg. Sci., $1: 247-52,2014$.

Pommer, B.; Tepper, G.; Gahleitner, A.; Zechner, W. \& Watzek, G. New safety margins for chin bone harvesting base don the course of the mandibular incisive canal in CT. Clin. Oral Implants Res., 19(12):1312-6, 2008.

Powell, N. B. \& Riley, R. W. A surgical protocol for sleep disordered breathing. Oral Maxillofac. Surg. Clin. North Am., 7(3):34556,1995 .

Sharma, N. S.; Shrivastav, S. S. \& Hazarey, P. V. Relationship among types of growth patterns, buccolingual molar inclination and cortical bone thickness of the mandible: A CT scan study. J. Ind. Orthod. Soc., 46:59-64, 2012.

Vu, D. D.; Brockhoff, H. C. 2nd.; Yates, D. M.; Finn, R. \& Phillips, C. Course of the mandibular incisive canal and its impact on harvesting symphysis bone graft. J. Oral Maxillofac. Surg., 73(2):258.e1-258.e12, 2015.

\author{
Dirección para Correspondencia: \\ Prof. Sergio Olate, PhD \\ CIMA \& CEMyQ Research Group \\ Division of Oral and Maxillofacial Surgery \\ Universidad de La Frontera \\ Claro Solar 115, Of. 414-A \\ Temuco \\ CHILE
}

Email: sergio.olate@ufrontera.cl

Recibido : 09-09-2014

Aceptado: 16-12-2014 